\title{
Beitrag zur Geschichte des Großen Aletschgletschers
}

\section{Einleitung}

«Zwo Stunden von Brig bey Naters ist ein neues Thal eröfnet welches oben von dem großen Anthonienberge beschlossen wird. Dieses Thal wird das Aletschthal genennet; es stürzt sich durch dasselbe der Massabach, mit vielem Gebrüel und in verschiedenen schönen Wasserfällen, über schrofe Felsen hinunter. Dieser Eisschrund ist ein beträchtliches Stück. Gegen Aufgang setzt er sich durch ein Stunden langes Eisthal zwischen den Bergen fort. Gegen Niedergang aber durchschleicht ein anderes Eisthal bis an die Gemmi die Vertiefungen des Berges fast ununterbrochen. $\mathrm{Da}$ wo dieser Eisschrund obenher Naters ausläuft, ist er sehr breit, und das Eis von ungemeiner Dichte, mit hoch aufgestellten Eiszacken versehen. (...) Nachdem er eine Stunde sich durch die Berge hindurch gedrängt, kommt er obenher Naters zum Vorschein, und fällt sehr anmutig in die Augen. Von Brig weg spielt er mit schönen Farben ineinander.»

Diese Worte hat G. S. GRUNER (1) in seinem 1760 herausgegebenen Werk Die Eisgebirge des Schweizerlandes> geschrieben. Der Eisschrund, der sobenher Naters ausläuft ist der Große Aletschgletscher, der jeden Sommer tausende von Wanderern in seinen Bann zieht. Heute ist dieser Gletscher von Brig aus nicht mehr sichtbar, da er sich seit dem letzten Hochstand um 1850 weit zurückgezogen hat.

Untersuchungen über Gletscheränderungen seit der letzten Eiszeit sollen mithelfen, die Klimageschichte zu erforschen. Am Geographischen Institut der Universität Zürich sind unter der Leitung von Prof. Dr. G. Furrer bereits verschiedene Arbeiten erschienen, und an einigen wird noch gearbeitet.

In diesem Artikel versuche ich, anhand einiger historischer Dokumente etwas über die Geschichte des Aletschgletschers auszusagen. Dabei geht es mir weniger darum, fertige Resultate zu liefern. Vielmehr möchte ich eine Methode zeigen, die zur Ermittlung früherer Gletscherausdehnungen und -stände als wichtiges Hilfsmittel herangezogen wird (Lit. 2, 3, 4): Historische Quellen, seien das nun Zeichnungen, Stiche, Gemälde oder schriftliche Aufzeichnungen. Wichtig ist, daß sich mittels eines solchen Dokumentes eine Aussage über den jeweiligen Zustand des Gletschers machen läßt. Dabei kann sich ein solches Dokument direkt auf den Gletscher beziehen (zum Beispiel Verwüstungen durch Gletschervorstöße, durch
Gletscherseeausbrüche oder ungangbar gewordene Pässe durch einen Gletschervorstoß). Häufig kann man jedoch nur indirekt Schlüsse auf die jeweilige Gletscherausdehnung ziehen (zum Beispiel durch alte Wasserleitungen, die einst Gletscherwasser führten [Lit. 5,6] oder durch einst begangene Wege, die ein Gletscherhoch- bzw. -tiefstand ermöglichte).

$\mathrm{Da}$ hier dieses Teilgebiet der Geographie aus Nachbargebieten Informationen schöpft, bewegt es sich im Interdisziplinären. Es zeigt sich, daß Geographie nicht nur als Erdbeschreibung im engeren, althergebrachten Sinne verstanden werden soll.

Bei der Suche nach historischen Dokumenten spielt oft auch der Zufall eine Rolle. Doch verspricht schließlich nur systematisches Suchen Erfolg. Ein bisher unentdecktes oder wenig beachtetes Schriftstück kann neue Aspekte liefern und die bisherigen Vorstellungen in ein neues Licht rücken. Im folgenden soll aufgezeigt werden, wie sich Bildmaterial und schriftliche Aufzeichnungen in besonders glücklichen Fällen gut ergänzen können.

\section{Ein alter Plan}

Bei der Suche nach historischem Dokumentationsmaterial den Aletschgletscher betreffend, stieß ich im Archiv von Sion (VS) auf einen alten Plan. Auf einem $67 \times 124 \mathrm{~cm}$ großen, auf Leinwand aufgezogenen Papier ist ein Teil des Aletschgebietes dargestellt (Abb. 1). Die Zeichnung wurde mit Tusche ausgeführt und schließlich koloriert. Unter der Bezeichnung «Plans divers No. 42» wird dieser Plan als Depositum der Gemeinde Ried-Mörel im Archiv aufbewahrt.

\subsection{Beschreibung des Planes}

Im Unterschied zu einer Landeskarte im Grundriß haben wir es hier mit einem Aufriß zu tun. Der Zeichner, dessen Name leider nicht bekannt ist, muß zur Anfertigung dieses Planes im Gebiet unterhalb Eggen seinen Standort bezogen haben mit Blick auf den Westabhang des Riederhorns. Dieses tritt als höchste Erhebung im oberen Bildteil in Erscheinung. Unten ist

Hanspeter Holzhauser, Assistent am Geographischen Institut der Universität Zürich, Blümlisalpstraße 10. 8006 Zürich. 


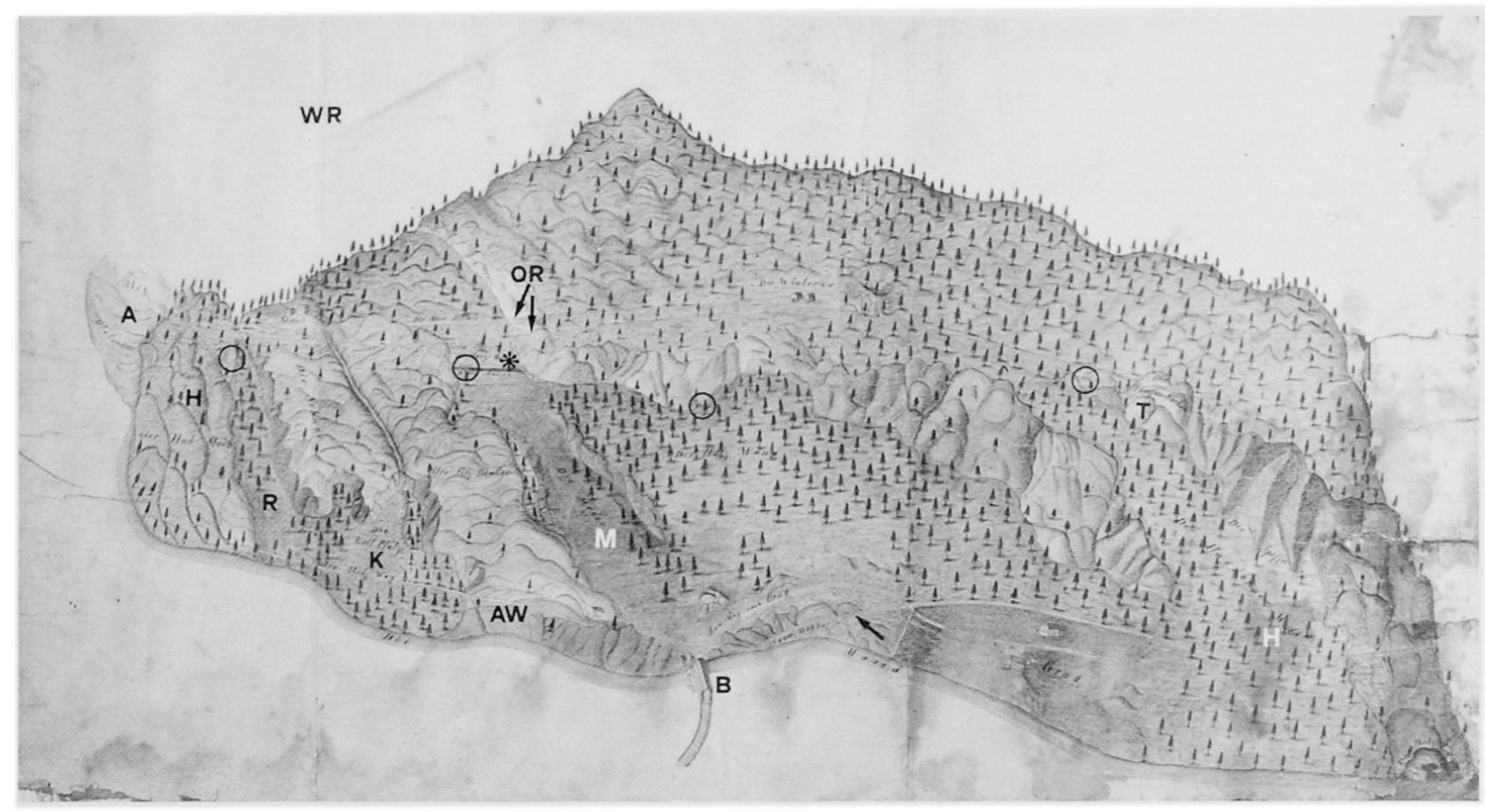

Abb. 1 Der alte Plan (Kantonsarchiv Sion, VS).

Die Kreislein umrahmen Jahreszahlen: $1756,17 \mp 56, \Psi 1755,1\lceil 56$ (v. I. n. r.).

\begin{abstract}
A Zunge des ‘Alez Gletschers $\quad$ H Hoch Stock (Stockflüe)
M Meder (Mäder) H Hagdorner Wald T Tapffer Nacken

R Rufigraben K Koll Platz (Kohlplatz) AW Aletschweg Die Pfeile deuten auf Wasserleitungen hin

OR alte Oberriederi

B Die Brugge * Terra jlloru de Morgia, 17 $\$ 56$, ein Mauren WR mit Bleistift eingezeichnete Windrose.
\end{abstract}

das Gebiet durch die Massa, den Abfluß des Großen Aletschgletschers, begrenzt. Da der Blick gegen Osten gerichtet ist, erscheint die Zunge des «Alez Gletschers» im Norden. Die Fallinie vom Riederhorngrat zum sog. Mutzloch〉 bildet ungefähr den südlichen Abschluß. Die vielen stilisierten Tannen deuten auf eine starke Bewaldung dieses Gebietes.

Als Orientierungshilfe wurde mit Bleistift schwach eine Windrose eingetragen. Diese befindet sich in der linken, oberen Ecke und trägt die Bezeichnungen «Morgen-Mittag-Abend-Mitternachts.

Will man das auf dem Plan dargestellte Gebiet in die Landeskarte übertragen (Blatt 1269, 1:25000), so erstaunt, wie gut dies gelingt - nicht zuletzt deshalb, weil der Zeichner anscheinend sehr bedacht war auf das genaue Eintragen von Lokalbenennungen. Auch die Topographie, markante Gräben und Felspartien, sind in einer Art festgehalten, die den Vergleich mit der neuen Landeskarte sehr erleichtert (vgl. dazu Abb. 2). Besonderes Interesse erwecken die Wasserleitungen und Wege: Erstere sind durch ausgezogene Linien, letztere gestrichelt dargestellt. Zwei Wasserfuhren ziehen unterhalb der Nessel〉 durch bis zu den Hütten von Die Winterna, wo sie zusammenlaufen und als gemeinsame Wasserleitung weiterführen.

Die obere Leitung trägt den Namen $\$ Die Ober Riederi). Ein weiterer 〈Aqua ductus〉 nimmt seinen Anfang bei der 〈Brugge〉, kreuzt den (Weg nach Grag) und verläuft oberhalb der Häuser von «Grag» Richtung Süden.

Ein Pfad, der wie der (Weg nach Grag) bei der Brücke über die Massa beginnt, wird im Plan als Der Aletz Weg〉 bezeichnet und führt am 〈Koll Platz〉 vorbei zum Gletscher. Es handelt sich dabei um den alten Aletschweg, der bei Hochständen des Gletschers über das Eis weiterführte (Abb. 3).

O. LUETSCHG (7) bemerkte dazu (p. 208):

«Der ehemalige Alpweg von Naters nach Aletsch führte die Hirten mit ihrem Vieh über die Gebidembrücke und dem hinteren Massaufer entlang bis zum sogenannten 〈Kohlplatz), $740 \mathrm{~m}$ aufwärts der Gebidembrücke. Von dieser Stelle aus folgte man dem Gletscher bis nach der Alp Aletsch.»

\subsection{Die Frage nach dem Alter des Planes}

Da auf der Darstellung die Zunge des Großen Aletschgletschers noch sichtbar ist, läßt sich über die Ausdehnung zur Zeit der Aufnahme etwas aussagen. Aus diesem Grunde ist die Enstehungszeit des Planes von Bedeutung.

Seine Existenz ist in der Arbeit von K. A. MEYeR (8) erwähnt. Dieser schreibt von einem noch nie veröffentlichten Plan aus dem 16. Jahrhundert. Die Kenntnis davon verdankt er Kantonsforstinspektor Perrig. MEYER beschreibt im folgenden diesen Plan, verlegt aber des- 


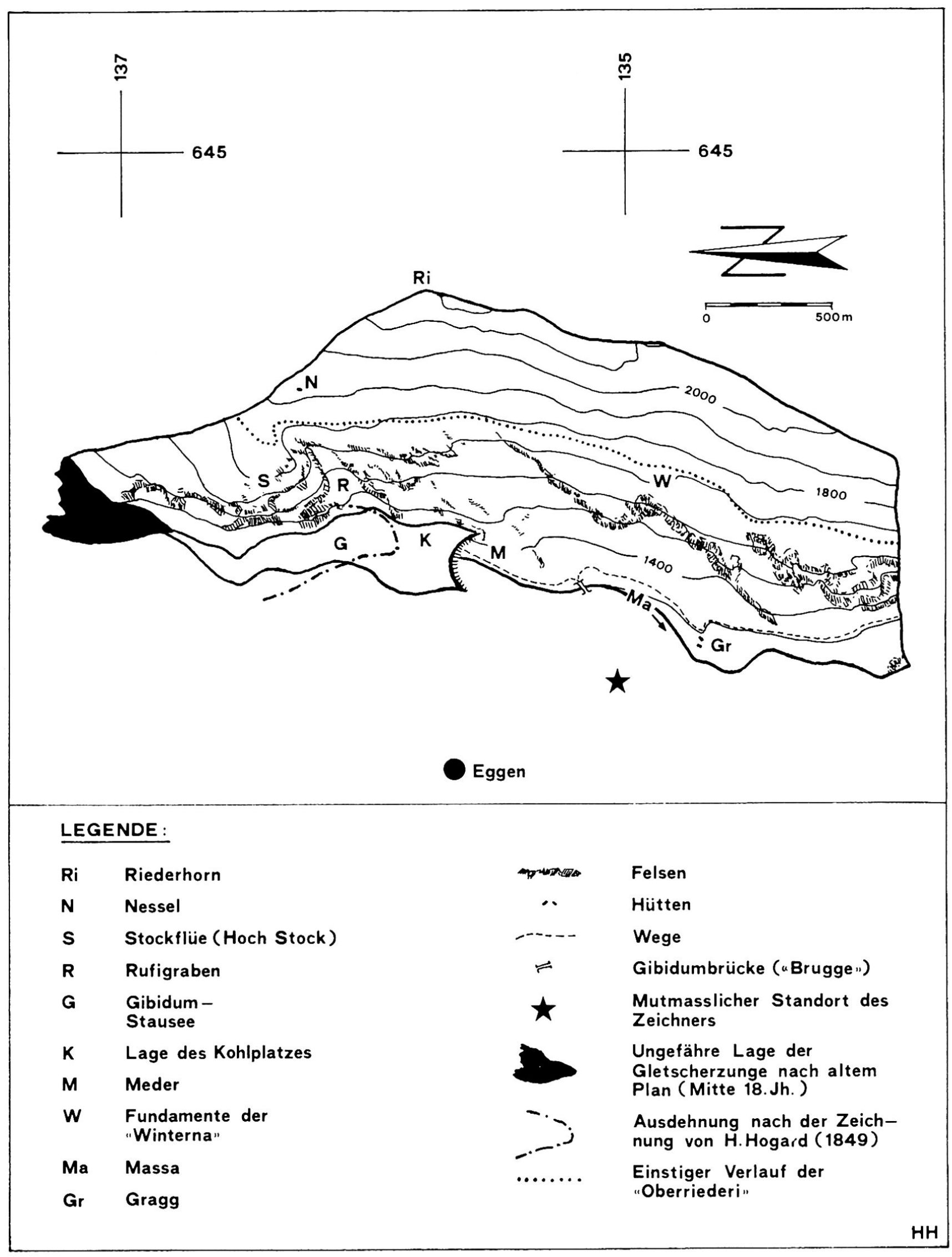

Abb. 2 Ausschnitt aus der Landeskarte, Blatt 1269 (1:25 000), der mit dem Plan vergleichbar ist. 


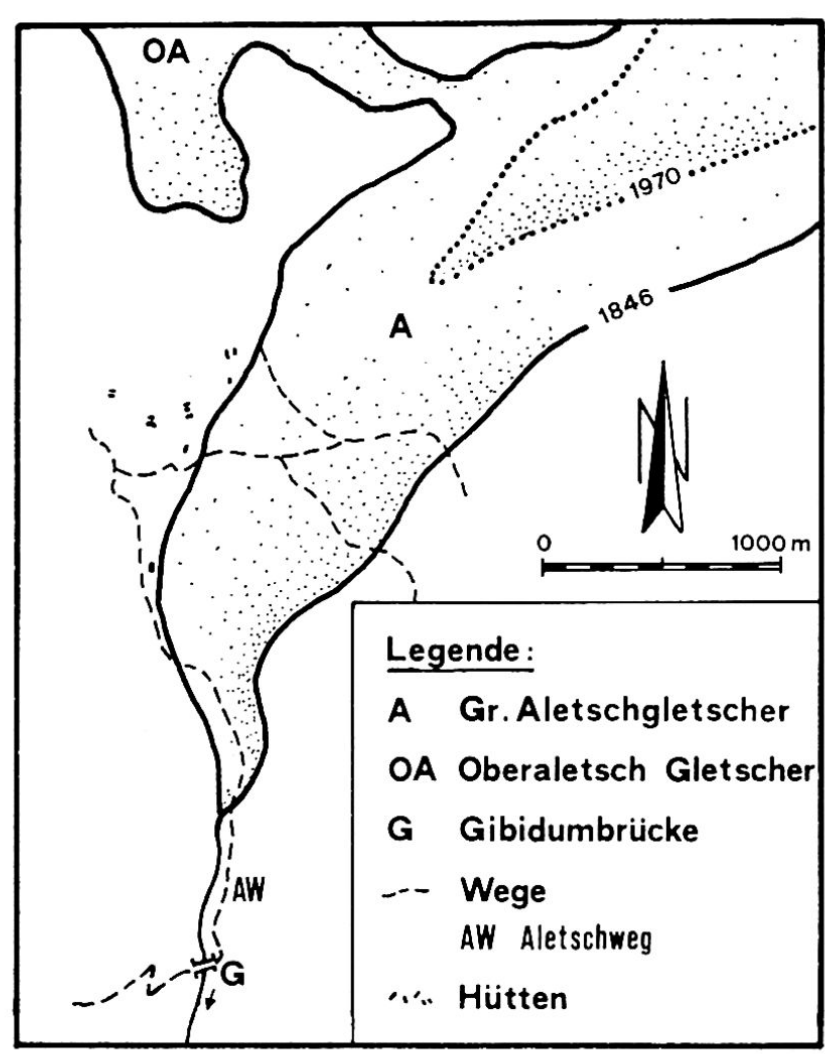

Abb. 3 Vergleich der Gletscherausdehnung von 1846 (Meßtischblatt MÜLLER) und derjenigen von 1970 (LK, Blatt 264,1:50 000). Eingetragen sind die Wege, die bei einem Hochstand über den Gletscher führten.

sen Herkunft in die zweite Hälfte des 17. Jahrhunderts. Anlaß dazu gibt ihm ein Schriftstück aus dem Jahre 1684 (vgl. Kap. 2.2.1.)

I. MARIETAN (9) weist in seinem Artikel über die Wasserleitungen ebenfalls auf dieses Dokument hin (p. 130): «Pour le versant ouest du Riederhorn les restes de murs sont assez nombreux, nous avons un plan aux Archives cantonales. La date est malheureusement effacée, on pense qu'il doit remonter au $18^{\mathrm{e}}$ siècle.»

\subsubsection{Schriftliches Quellenmaterial}

Vom 31. Mai 1684 datiert ein Schriftstück (Bitsch, C 10), worin das auf dem Plan dargestellte Gebiet beschrieben wird: «Das Dekret des Landeshauptmann Stockalper vom 8. August 1677 wird bekräftigt. Die Burgschaft Naters und die Gemeinde de Richinen sollen trotz des Entscheides von 1683 im Besitze der Weidgänge und Allmeinen jenseits der Massa gegen Mörel bleiben, nämlich der Mäder, der Sitz Blatten, des Holz Wang, des Hegdorner Waldes, der Spissa, des Aletsch Waldes, des Kohll Platzes, des Scheuers, der Ruffina, welches alles morgenhalb grenzt an den Hoch Stock und an das Land der Mörier zu oberst an den Mädern einen Steinwurf über sich unter der alten Oberriederin, mittags an den Tapfernacken, abends an das Mutzloch, mittnachts an die Massa aus dem Aletschglet- scher mit Weg, Wald, Eigen- und Gemeindegüter, Viehweiden und Allmeinen. Das von Ried und Bitsch erhaltene Dekret von 1683 wird als ungültig verworfen, die Massa als Zehndenmarch anerkannt. Die Prozeßkosten haben beide Parteien zu tragen oder der Viertel Ried allein, wenn er sich weigert, dieses Urteil anzunehmen, mit 3 Dubeln Strafe für die Vermessenheit.»

Dieses Dokument beinhaltet ein Gerichtsprotokoll über die Besitzverhältnisse zwischen Naters, Richinen, Mörel, Ried und Bitsch. Es erweckt den Eindruck, daß über die Zugehörigkeit gewisser Güter unter den Gemeinden Uneinigkeit herrschte. Diese Vermutung wird durch weitere, früher verfaßte Urkunden bekräftigt. So hat jene vom 18. Januar 1684 folgenden Inhalt (Pfarrei Naters, C 16):

«Fragepunkt der Gemeinde Ried und Bitsch von Herrn Landshauptmannstatthalter über die durch Naters ausgeführte Viehpfändung und ob Naters die Massa als Zehndenmarch erkenne.»

Am 13. April 1684 erfolgte die Antwort von Naters (Pfarrei Naters, C 17):

«Protest der Burgschaft Naters, die Massa als Zehndenmarch erkennen zu müssen, weil sie vom Zehnden nicht beauftragt worden ist.»

Daraufhin wurde das Urteil vom 31. Mai 1684 geschrieben (siehe oben). Daß die Gemeinden Ried und Bitsch schon im Jahre 1626 Land in der Gegend der Nessel besessen haben, geht aus einem Dokument vom 26. Februar 1626 hervor:

«Die Gemeinden Bitsch und Ried verkaufen dem Martin Berchtold Allmeine und Wald im Nessel, im rothen Bruch und im Klosstein; unter Angabe der Grenzen, ob der alten Wasserleite und dem Weidgang der Naterser mit Vorbehalt der grünen Lärchbäume, welche der Käufer und sein Nachfolger noch unterhauen, schwenden noch erdörren dürfen, und daß das Vieh dieser Gemeinden darauf nicht gepfändet, sondern nur friedlich abgetrieben werden darf.» Auf die Urkunde vom 31. Mai 1684 stützt MEYER seine Vermutung, der Plan sei in jener Zeit erstellt worden. Die Auseinandersetzungen unter den einzelnen Gemeinden brechen aber mit dem Jahre 1684 nur vorläufig ab. Siebzig Jahre - bis 1754 - scheint in dieser Angelegenheit Ruhe geherrscht zu haben. Möglich ist auch, daß aus dieser Zeitspanne keine Dokumente mehr existieren.

Verfolgen wir die Geschichte weiter:

Am 11. und am 12. September 1754 (Ried-Mörel, C 30) wurde ein Gerichtsprotokoll «bei dem Augenschein über das Zeugenverhör der Naterser bezüglich Besitz der Marchen in den Mädern' aufgenommen, und zwar bei der $\langle$ Bidembrück $\rangle$ ).

Streitpunkt in den darauf folgenden Verhandlungen scheinen die «Mäden» zu sein (vgl. Abb. 1 und Abb. 2). Es herrscht wieder einmal Uneinigkeit zwischen Ried, Bitsch und Naters. Vom besagten Zeitpunkt an (11.September 1754) bis zum 21. April 1755 wird prozessiert. Davon zeugen folgende Akten (Ried-Mörel, C 31-35): 


\section{1.-27. November 1754 (C 31):}

Gerichtliches Zeugenverhör zugunsten von Ried und Bitsch gegen Naters über Eigentum und Rechte in den Mädern.

\section{? Januar 1755 (C 32):}

Memorial des Viertels Ried gegen das Zeugenverhör der Naterser und ihre Belegstücke über die Rechte in den Mädern.

\section{Februar 1755 (C 33):}

Gegen-Memorial der Naterser über die rechtlichen Einreden der Rieder gegen ihr Zeugenverhör, rechtliches Vorgehen und die Grenzen der Mäder.

\section{April 1755 (C 34):}

Gerichtliches Urteil im Handel der Gemeinde Ried und Bitsch gegen Naters bezüglich der Mäder. Diese gehören Naters; es werden die Grenzen beschrieben. Naters soll den Riedern das zum Unterhalt ihrer Wasserleite nötige Holz verabfolgen lassen.

\section{April 1755 (C 35):}

Protest der Naterser-Geteilen in den Mädern für ihre Rechte in den Mädern und Ergänzung der Marchen.

Beim genaueren Betrachten des Planes erkennt man vier Jahreszahlen (vgl.Abb.1): 1756,17 456 , ↔ 1755 und 1Г56. Bei der letzten Jahreszahl ist die Ziffer (7) verkehrt eingetragen. Die Bezeichnung (Terra jlloru de Morgia, 17千56, ein Mauren > ${ }^{2}$ deutete MEYER als «nachträglich beigefügten Titel». Meiner Meinung nach ist dies nicht zutreffend, da mit dieser Beschriftung Güter, die Mörel gehören, angedeutet werden. In diesem Sinne ist auch (Der Hagedorner Wald zu verstehen (Hegdorn befindet sich in der Nähe von Naters). Von den oben erwähnten Jahreszahlen erwähnt MEYER jedoch nichts. Diese haben vermutlich eine enge Bewandtnis mit dem Entstehungsdatum des Planes. Ebenso muß ein Zusammenhang zwischen dem Prozeß von 1754/55 und dem Plan bestehen.

Licht in diese Angelegenheit bringen Prozeßakten aus den Jahren 1855/56, als erneut ein Streit unter den Gemeinden entbrannte.

In einer Anfrage seitens Naters an das Gericht am 27. September 1855 (Gemeinde Naters, C 16) geht hervor, daß über die Grenzverläufe im Gebiet der «Mäden keine Einigkeit herrschte. Dabei ist von Marchen die Rede. Es handelt sich bei diesen um die auf dem Plan eingetragenen Jahreszahlen. Naters stützt sich in ihrem Schreiben auf das Urteil vom 12. April 1755 (siehe oben) und auf den Plan. Unter anderem heißt es in der Anfrage Naters:

«1. Die im Akte oder Urtheil vom 12. April 1755 als Ausgangspunkt angenommene Mark vom Hochstock oder Wandstock finde sich wirklich an der im natischerseits vorgelegten Plane oder Abrisse als Wand- oder Hochstock bezeichneten Stelle vor und sei von den Parteien beidseitig anerkannt.»
Unter Punkt 7 steht:

$« \mathrm{Daß}$ am obersten Fuße der im Abrisse als Tapfernacken angezeichneten Felse die Jahreszahl 1756 eingeschnitten ist ungefähr in der gleichen Entfernung von der Oberriederi als die Mark ob den Mädern.» Das Gericht, nach vorgenommenem Augenschein, fällt am 10. Januar 1856 das Urteil in diesem Streit. Als Antwort zu Punkt 7 von Naters schreibt es:

«Am oberen Fuß des auf dem Plan bezeichneten Tapfernackens steht wirklich die gleiche Jahreszahl wie auf der Mark ob der Medern, und zwar in einer nicht viel größeren Entfernung von der Oberriederi, als jene ob den Medern, nur ist die Ziffer 7 umgekehrt /: $1\lceil 56$ :/ und steht kein Kreuz in der Mitte.» Ebenso Bezug nimmt das Gericht auf die von Ried und Bitsch (die Gegenpartei) eingereichten Fragen. In der Stellungsnahme des Gerichtes werden die Marchen genau beschrieben, was aus der Antwort zur Frage 1 dieser Gemeinden hervorgeht:

«Die Mark ob den Mädern, tragend die Jahreszahl 1756 , ist von beiden Parteien anerkannt, sie liegt linkerseits eines Brünleins, 13 Klafter unter der Oberriederi und $86 \mathrm{Klafter}$ von dem von der Parthey Ried angerufenen Felsbord.»

Aus obigen Ausführungen geht klar hervor, daß es sich bei den besagten Jahreszahlen um Marchen handelt, die an den betreffenden Stellen in den Fels eingehauen sind.

Auf Grund dieser Tatsache bin ich der Überzeugung, daß der Plan nicht vor 1755 angefertigt wurde. Es ist kaum wahrscheinlich, daß man den Plan vor dem Einhauen der Marchen in den Felsen gezeichnet hat. Eher ist es umgekehrt. Der Aufriß ist im Anschluß an das Gerichtsverfahren von $1754 / 55$ erstellt worden, um die Güter, Grenzen und Marchen so optisch fixieren zu können. Der Zweck dieses Dokumentes ist in diesem Sinne zu verstehen.

Was läßt sich nun über den Gletscher aussagen?

Der Plan zeigt uns den Großen Aletschgletscher zu Beginn der zweiten Hälfte des 18. Jahrhunderts. Die Gletscherzunge lag wesentlich weiter zurück als rund hundert Jahre später, nämlich um 1850 (siehe Kapitel 3). Die Ausdehnung entspricht etwa derjenigen gegen Ende des 19. Jahrhunderts. Auch im Jahre 1778 war der Gletscher vermutlich kleiner als bei einem Hochstand. Dies geht aus einer Beschreibung von G. S. GRU. NER (10) hervor (p. 220, 221):

«Nach zwey Stunden kam ich über die Alpen Hagdorn, Blatten und Bellalp nahe an die Gletscher ${ }^{3}$. Als ich etwas näher kam, fand ich dieselben wie mit Wällen eines Feldlagers, und von Menschen Händen aufgeworfene Redouten gleichsam verschanzt. (...) Ich gewahrte verschiedene dieser Redouten an Orten, die dermalen ziemlich weit von den Gletschern entfernt waren.»

Die Moränenwälle, die GRUNER hier beschreibt, markieren frühere Gletschervorstöße. Um welche Vorstöße es sich hier gehandelt hat, kann nicht mit Sicherheit gesagt werden. 


\section{Bilddokumente}

Im August 1849 reiste H. HOGARD ins Tal der Massa. Glücklicherweise hielt er die Situation bei der Gletscherzunge in Form eines Aquarells, das später lithographiert wurde, fest (Abb. 4, Lit. 11). Eine zweite, skizzenhafte Zeichnung zeigt, wie der Gletscher den alten Aletschweg teilweise zerstört (Abb. 5, Lit. 12). HOGARD (13) bemerkt dazu (p. 316):

«Envahissement du glacier d'Aletsch à sa pente terminale. En 1849 il continuait à couper la chaussée, dite le chemin des Maures, et à s'avancer vers un groupe de sapins qu'il a renversés depuis cette époque.»

Bei DOLLFUS-AUSSET (14)ist in Zusammenhang mit der Zeichnung HOGARD's zu lesen:

«Le glacier d'Aletsch, et sa pente terminale, rive gauche. En 1849, ce glacier était en voie rapide de progrès dans la partie supérieure; il avait fermé l'accès des châlets d'Aletsch, habités il y à peu d'années encore. Il s'avançait dans la forêt, recouvrant des sapins séculaires; notre croquis représente sur la rive gauche l'ancien chemin de la haute vallée fermé et complétement détruit à partir de ce point.»

$\mathrm{Da}$ der Große Aletschgletscher im Vorrücken war, geht aus den Darstellungen von HOGARD sehr schön hervor. Deutlich sieht man in Abb. 4 den Moränenkranz um die Gletscherzunge. Die umgeworfenen Bäume sind ebenfalls Zeugen des mächtigen VorstoBes. Da Dollfus-AUSSET von "sapins séculaires», also von mehrere hundert Jahre alten Tannen spricht, ist anzunehmen, daß der Gletscher seit etlicher Zeit nicht diese Ausdehnung erreicht hat. Eine Parallele läßt sich auch zum Fieschergletscher ziehen. Dort stieß der Gletscher um 1850 in den Wald vor und knickte 200bis 300jährige Rottannen (6, p. 55).

Vermutlich hatte der Große Aletschgletscher um 1653 eine ebensolche, wenn nicht größere Ausdehnung. Aus jener Zeit wird nämlich von einer Prozession berichtet, die den allzu kräftigen Gletschervorstoß abzuwenden versuchte.

Über den Hochstand von 1850 schreibt LUETSCHG (7): «Nach den Aufzeichungen von Kaplan Bamatter von Naters und in Übereinstimmung mit vielen Talbewohnern hatte der Gletscher seinen höchsten Stand im Jahre 1856 erreicht. Anno 1875 kam der Gletscher noch bis zum Kohlplatz, hernach ging er kontinuierlich zurück. 1886 wurde das Vieh zum letzten Male über den Gletscher geführt.»

Im Zusammenhang mit dem Kohlplatz〉 ist das Dokument vom 31. Mai 1684 (Bitsch, C 10; vgl. Kapitel 2.2.1.) von Interesse. In diesem Schriftstück werden u.a. der 〈Kohlplatz〉 («Kohll Platz〉) und der (Rufigraben (〈Ruffina )) als Grenzen angegeben. Beim Hochstand um 1850 lag aber die Gletscherzunge auf diesem Platz, ebenso auf dem unteren Teil des (Rufigrabens). Wenn das 1684 auch der Fall gewesen wäre, hätte man diese Lokalitäten nicht als Grenzen verwendet oder zumindest den Gletscher erwähnt.

An Hand der Beobachtung von DOLLFUS-AUSSET kann angenommen werden, daß der Große Aletschgletscher um 1820 kleiner war als um 1850, sonst hätte der Gletscher nicht so alte Tannen umdrücken können.

Ein Ausschnitt des alten Planes und die Lithographie von HOGARD (Abb. 4) lassen sich gut miteinander vergleichen: Der Rufigraben ist in der Darstellung von HOGARD rechts oben ersichtlich. Im unteren Teil ist dieser Graben mit Eis und Moränenmaterial verschüttet. Die Gletscherzunge reicht nahe an den sogenannten «Kohlplatz» heran, der teilweise im rechten unteren Teil des Bildes zum Vorschein kommt und durch welchen der Aletschweg führt. Im Plan ist dieser \& Rufigraben» und der 〈Kohlplatz〉 ( Koll Platz〉) ebenfalls gut erkennbar (Abb. 1.) Der erwähnte Graben zieht zwischen dem (Hoch Stock» und der 〈Schnun durch. Die Gletscherzunge aber liegt weit zurück (vgl. Kapitel 2.2.1.).

\section{Zusammenfassung}

Ein alter Plan konnte mit Hilfe von Dokumenten zeitlich eingeordnet werden. Besonders glücklich ist hier das Zusammentreffen von Bildmaterial und schriftlichem Quellenmaterial aus verschiedenen Epochen, die sich sinnvoll ergänzen. Zum Schluß möchte ich die einzelnen Aussagen nochmals kurz festhalten:

1. um 1684 war die Ausdehnung des Großen Aletschgletschers kleiner als um 1850 (geht aus dem Dokument vom 31. Mai 1684 hervor).

2. Wie dem Plan zu entnehmen ist, war der Gletscher zu Beginn der zweiten Hälfte des 18. Jahrhunderts wesentlich kleiner als um 1850. (Die Ausdehnung entspricht etwa derjenigen gegen Ende des 19. Jahrhunderts.)

3. Um 1780 lag der Gletscher hinter den Moränen eines früheren Vorstoßes (Beschreibung von GRUNER).

4. Vom Hochstand um 1850 zeugen zwei Bilddokumente von H. HOG ARD. Der Gletscher drückte mehrere hundert Jahre alte Tannen nieder.

5. Durch die Angaben von O. LUETSCHG wissen wir etwas über die Entwicklung des Gletschers nach seinnem Hochstand, der um 1856 beendet war. Bis 1875 war der Gletscher nicht wesentlich zurückgewichen (er kam noch bis an den Kohlplatz heran). Erst nach dieser Zeit fand der große Rückzug statt.

Abb. 4 Glacier d'Aletsch. Lithographie nach einer Zeichnung $\triangleright \Delta$ von H. HOGARD, 1849. Deutlich erkennt man den vorrückenden Gletscher, der mit seiner Stirn mächtige Tannen knickt. Rechts im Vordergrund ist der «Kohlplatz erkennbar. Oben rechts zieht der 'Rufigraben, unterhalb des 'Hoch Stockes' (Stockflüe) durch.

Abb. 5 Glacier d'Aletsch. Pente terminale, rive gauche. Der Gletscher zerstört den Aletschweg (links). Zeichnung von H. HOGARD, 1848. 


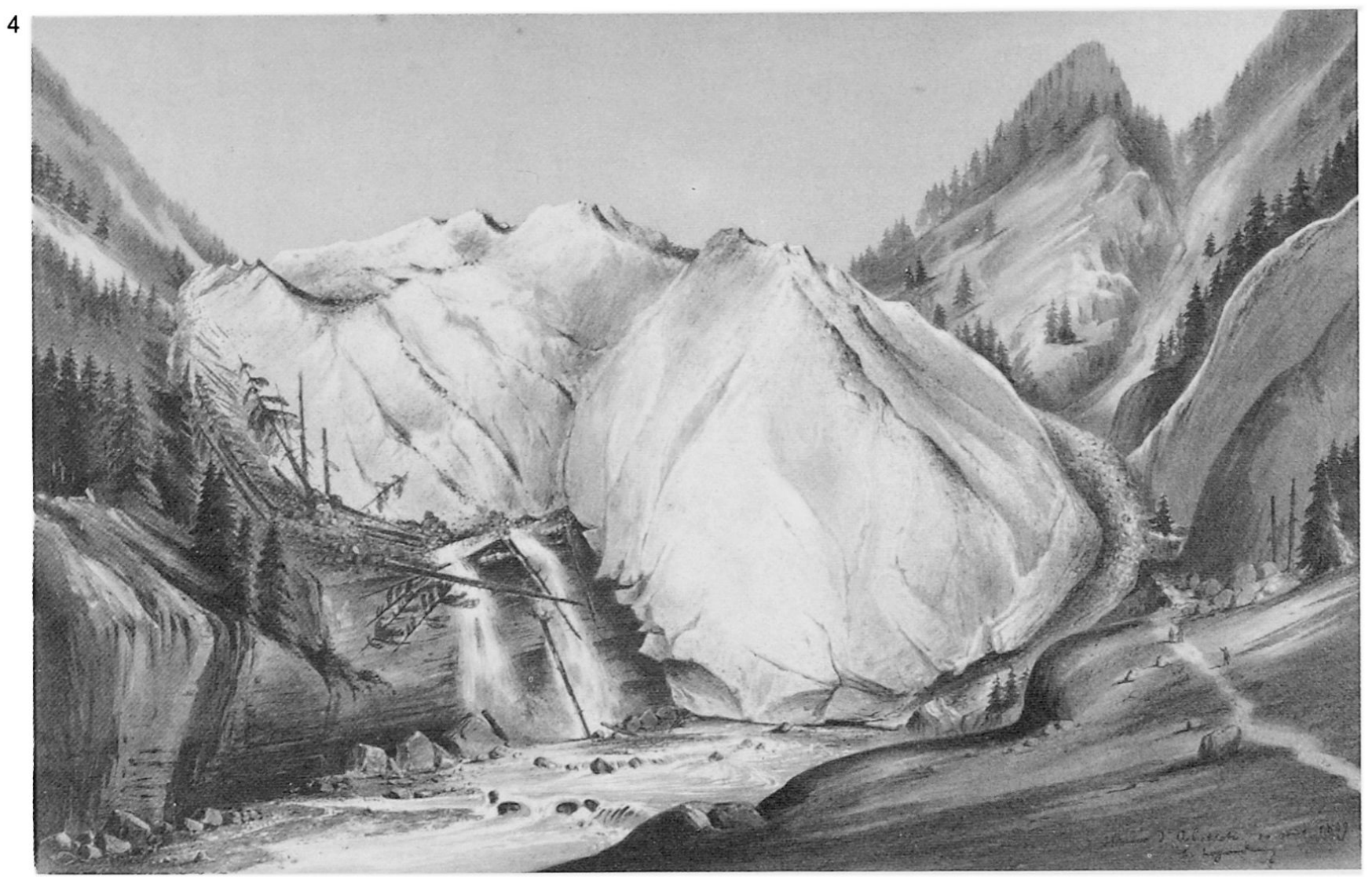

5

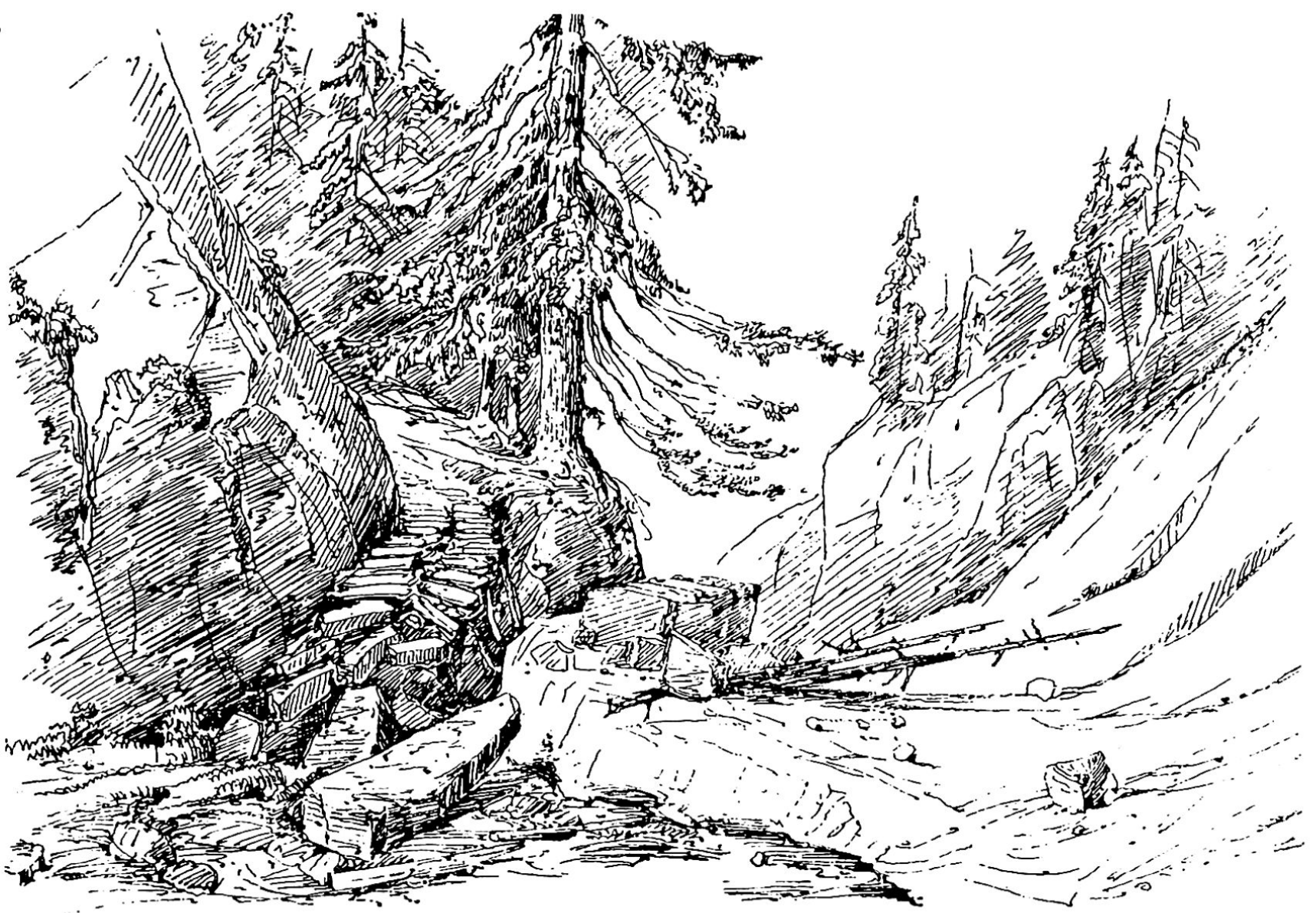

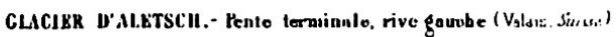




\section{Anmerkungen:}

1) Bidembrücke: = Gibidembrücke; auf dem Plan als Die Brugge bezeichnet

2) (Terra jlloru de Morgia): Land, das Mörel gehört (Morgia = Mörel). LEin Mauren bezieht sich auf eine Mauer.

3) GRUNER meint dabei den Oberaletsch Gletscher und den Großen A.letschgletscher, die beide über die Belalp gut erreichbar sind.

\section{Literaturverzeichnis}

(1) GRUNER, G. S. (1760): Die Eisgebirge des Schweizerlandes. 3 Bde.; Bd. I, p. 198, 199; Bern.

(2) KINZL. H. (1932): Die größten nacheiszeitlichen Gletschervorstöße in den Schweizer Alpen und in der Mont-Blanc-Gruppe.

Zeitschrift für Gletscherkunde, XX. Band, p. 269 bis 397, Leipzig.

(3) RÖTHLISBERGER, F. (1976): Gletscher- und Klimaschwankungen im Raum Zermatt, Ferpècle und Arolla. Diss., Geogr. Institut, Uni Zürich.

(4) ZUMBÜHL, H. J. (1975): Die Schwankungen des Unteren Grindelwaldgletschers in den historischen Bild- und Schriftquellen des 12. bis 19. Jahrhunderts.

Zeitschrift für Gletscherkunde und Glazialgeologie, Bd. XI, Heft 1, 1975, p. 12-50.

(5) OESCHGER, H., RÖTHLISBER GER, H. (1961): Datierung eines ehemaligen Standes des Aletschgletschers durch Radioaktivitätsmessung an Holzproben und Bemerkungen zu Holzfunden an weiteren Gletschern.
Zeitschrift für Gletscherkunde und Glazialgeologie, Bd. IV, Heft 3, 1961, p. 191-205.

(6) HOlZhAuser, H. (1978): Zur Geschichte des Fieschergletschers. Dipl.-Arbeit, Geogr. Institut, Uni Zürich.

(7) LUETSCHG, O. (1915): Der Märjelensee und seine Abflußverhältnisse. Annalen der Schweizerischen Landeshydrographie, Band I.

(8) MEYER, K. A. (1955): Frühere Verbreitung der Holzarten und einstige Waldgrenze im Kanton Wallis. IV. Oberwallis.

Mitteilungen der Schweizerischen Anstalt für das forstliche Versuchswesen, XXXI. Band, Heft 3, p. 563-668.

(9) MARIETAN, I. (1961): Observations sur les bisses de Moosfluh et d'Oberriederi.

Bulletin de la Murithienne, Fascicule LXXVIII, 1961, p. 128-130.

(10) GRUNER, G. S. (1778): Reisen durch die merkwürdigsten Gegenden Helvetiens. London, 2 Bände; Band I.

(11) DOLLFUS-AUSSET, D. (1872): Atlas. Enthält Lithographien nach Darstellungen von H. HOGARD (1848/49). (Zentralbibliothek Zürich.)

(12) HOGARD, H. (1858): Recherches sur les glaciers et sur les formations erratiques des Alpes de la Suisse. Planches. Paris. (PL. 20, Fig. 1).

(13) HOGARD, H. (1858): Recherches sur les glaciers et sur les formations erratiques des Alpes de la Suisse. Epinal.

(14) DOLLFUS-AUSSET, D. (1872): Matériaux pour l'étude des glaciers. Explications des planches (Atlas zum 8bändigen Werk). Strasbourg. PL. 40, Fig. 4. 